\title{
Coherent State Operators in Cosmology and Gravity
}

\author{
Andrea Dapor* \\ Faculty of Physics, University of Warsaw \\ E-mail: adapor@fuw.edu.pl
}

Coherent States (CS) are widely used in physics, and quantum gravity is not an exception. However, the application of CS in the construction of operators is rather unexplored in the areas of quantum gravity and quantum cosmology. In my talk, I will present how CS can be used to define "coherent state operators" via a procedure known as "coherent state quantization" [1]. This procedure produces operators with inbuilt good semiclassical properties, while at the same time preserving typical quantum attributes (e.g., discreteness of spectra). Examples of such coherent state quantization will cover: (1) operators on homogeneous isotropic quantum cosmology [2] (based on the affine group); (2) possible extensions to Bianchi I case; (3) simple operators on $L_{2}\left(S U(2), d \mu_{H}\right)$.

References

[1] S. Twareque Ali, J.-P. Antoine and J.-P. Gazeau, Coherent States, Wavelets, and Their Generalizations, Springer-Verlag (2013)

[2]H. Bergeron, A. Dapor, J.-P. Gazeau and P. Malkiewicz, Smooth big bounce from affine quantization, Phys. Rev. D 89, 083522 (2014)

Frontiers of Fundamental Physics 14 - FFP14,

15-18 July 2014

Aix Marseille University (AMU) Saint-Charles Campus, Marseille

${ }^{*}$ Speaker. 